\title{
SOME RADIOGRAPHIC MANIFESTATIONS OF EARLY SCURVY
}

\author{
BY \\ JAMES F. BRAILSFORD \\ From the Royal Orthopaedic Hospital, Birmingham
}

(RECEIVED FOR PLBLICATKON OCTOBER 28, 1952)

Though scurvy is associated with characteristic radiographic appearances it is important to realize that it can manifest itself clinically before radiography detects any change from the normal in the bones. The latent negative radiographic period may be of several months. In most conditions it varies with the individual and the factors which are associated with the deficiency. There is some evidence that the deficiency factors, as in rickets, act upon the foetus in utero and in the early days of separate life. though scurvy is commonly regarded as a deficiency disease which shows itself between the eighth and eighteenth months. The initial lesion which usually attracts clinical attention is the haematoma. Hutchinson and Moncrieff (1941) have pointed out that

the chief changes in infantile scurvy are in the neighbourhood of the bones. A section made across a limb at the site of a swelling shows that the periosteum is hypervascular, thickened and separated from the subjacent bone by a laver of partially organized blood clot. There is no sign of inflammation and no hard bone is formed in the periosteum except in longstanding cases."

Therefore, in these early cases we see no sign of change in the bones. The first radiographic evidence may be a subperiosteal haemorrhage (Fig. 2) or fractures at the growing extremity of one or more of the long bones (Fig. 1), but usually such lesions have been present for a week or more before radiographic examination has been made, for we often see that already some calcium has been deposited in an amorphous form within the associated haematoma. and is most evident at the periphery of the haematoma, leaving a translucent zone between it and the periphery of the shaft of the bone (Fig. 2).

Infants, apparently healthy in other respects, have been seen at birth with considerable calcium in haematomata associated with fracture, indicating intra-uterine damage of some weeks' duration. Massive haematomata with much calcium surrounding both femora of an unusual infant at birth have been illustrated (Brailsford, 1948). Certainly, within the first few months of life radiographs may show amorphous calcium deposition in haematomata enveloping one or more long bones, which appear to have normal structure and density, and, though no other definite indication of scurvy may be present, organization, ossification and complete restitution to normal bone takes place readily on the administration of vitamin C. These features are illustrated by the sequence of radiographic changes in Fig. 2 .

Fig. 2 illustrates the case of an infant, aged 3 months, who developed a swelling in the left thigh. A radiograph of the thigh on August 15, 1947 (Fig. 2A), showed amorphous calcium deposited in a large subperiosteal haematoma which enveloped most of the shaft of the femur. A further radiograph (Fig. 2B) on August 28, 1947, showed an increase in the size of the lesion, and its definition suggested that rapid organization and ossification was taking place. The radiograph of September 8, 1947 (Fig. 2C), shows that within a month most of the haematoma had been converted into bone. On the clinical and radiographic evidence this was interpreted as sarcoma and drastic treatment was contemplated. The films were submitted to me by Dr. Patricia Franklyn, and I suggested that the condition was due to scurvy and advised the administration of appropriate amounts of vitamin $\mathrm{C}$. With its exhibition the patient rapidly showed signs of recovery and by January 8 , 1948 (see Fig. 2D), considerable resolution had taken place. By 1952 the bone had returned to normal (Fig. 2E). Obviously in this case the clinical signs of scurvy were not present and the radiographic evidence had been misinterpreted but the therapeutic test was both positive and spectacular.

In 1943 in a paper on osteogenesis imperfecta, I published an account of four patients with this dystrophy who developed an unusual complication, namely, the development around one or more bones of a swelling. probably as the result of trauma, since it was observed that a similar sequence of changes 


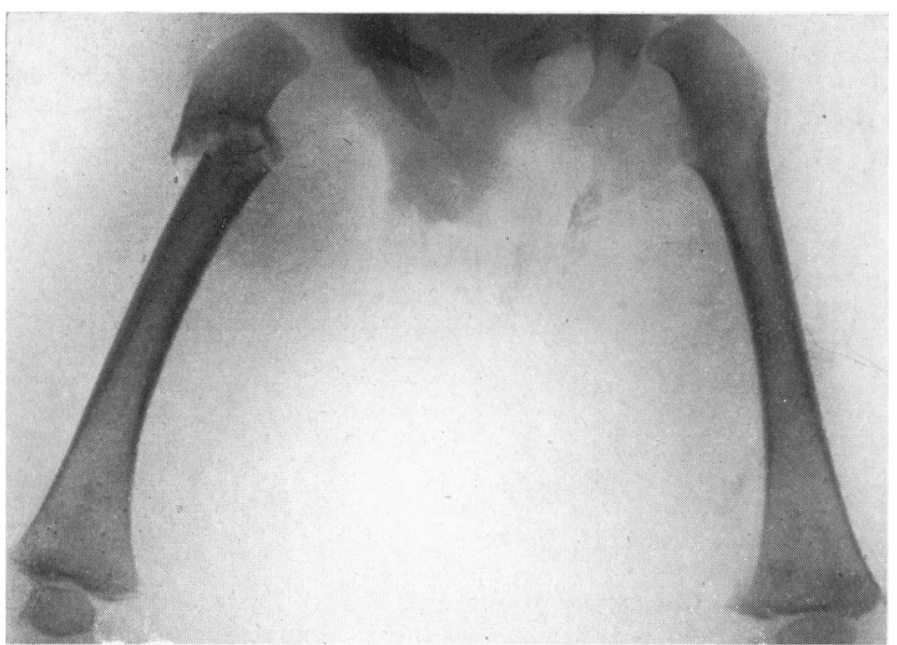

A.-On admission on January 19, 1948, fractures at the lower ends of the femoral diaphyses and a subtrochanteric fracture of the upper third femur are seen.
FIG. 1.-Radiographs of limbs of an emaciated and neglected infint aged 11 months.

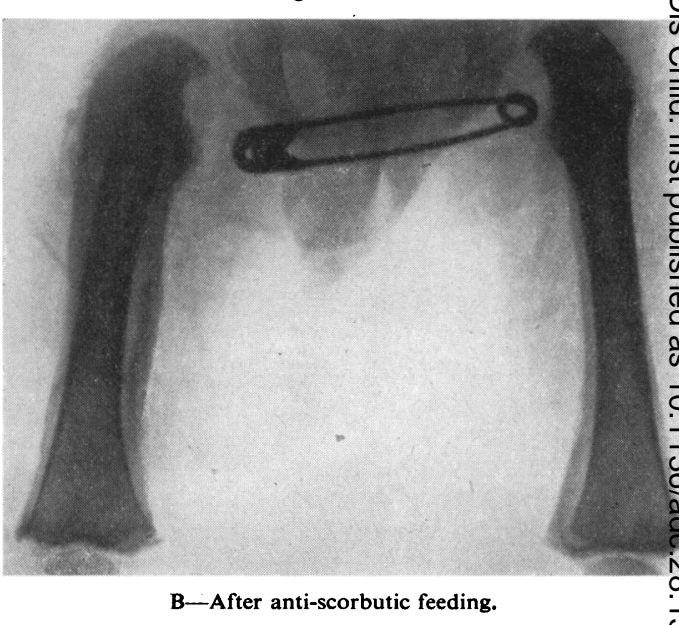

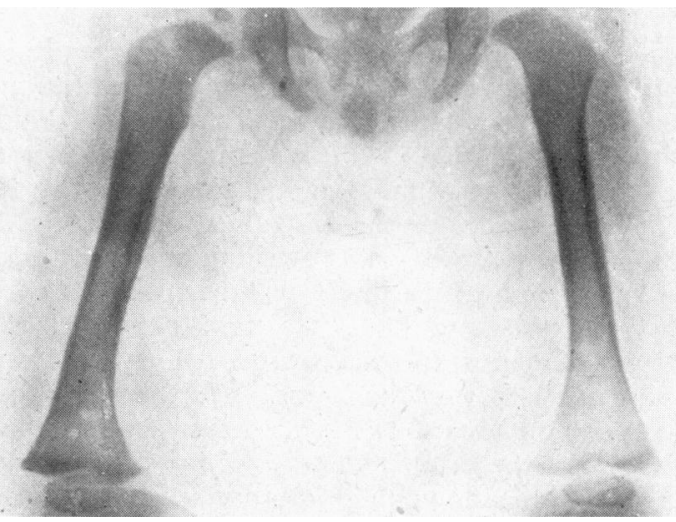

C-Almost complete resolution of haemorrhages.

took place at the site of surgical injury. The trauma was such that it escaped the attention it demanded. Radiographs of the recent swelling of the inner side of the right femur showed the rounded outline of a large, soft-tissue mass. Its almost sudden appearance, its extent and its clearly defined rounded border indicated that it was probably a haematoma. Other tumour masses in the same and adjacent limbs suggested masses of longer duration presenting the appearances which, after a sequence of changes, developed in the most recent (Fig. 3A). The serial radiographic appearances of these lesions were identical with those seen in haematomata, that is, large, rounded, soft-tissue swelling around the bone of an acute haematoma which gradually took on a cloudy and later a granulated appearance. The greater density indicated calcium deposits in the haematoma but in this condition the calcium deposit is denser than in haematomata associated with normal bone. With the passage of time organization of the calcium and ossification was indicated by the diminishing mass and the corrugated appearance of its shrunken periphery, and gradually by the definition and density of the bone. The new bone persisted in its irregular form and the details of the enveloped femoral shaft were completely absorbed (Fig. 3B).

This is a very unusual sequence of changes for osteogenesis imperfecta, in which are usually seen fractures which heal without any undue callus forma tion or around which there may be little evidence of callus, the fragments remaining disunited for many? years or even throughout a subsequent long life. There is only one other condition which resembles it, the haematoma which forms at the site of a fracture of a bone of a paralysed limb of an otherwise normal skeleton. I have no doubt about the haematoma being due to injury in such cases, and the resulting bone deformity does not undergo the ready moulding seen in the normal limb (Figs. 4A and B) but there was no indication of paralysis in these patients.

I was led to consider the factor which could be responsible for multiple haemorrhages on relatively slight trauma, and the possibility of the added complication of scurvy suggested itself. Scurvy is the only condition, apart from haemophilia and leukaemia and other conditions, with their characteristic clinical signs and symptoms, in which are seen multiple sites with subperiosteal haematoma around bones which may be apparently normal radiographically. But in scurvy the haematomata are readily organized on the administration of vitamin C. They follow a definite sequence of changes. They calcify and become ossified, the new bone is gradually absorbed, and finally the affected bone becomes and remains indistinguishable from the normal in 
shape and size and structure (Figs. 1 and 2). In osteogenesis imperfecta we see the initial stages as far as ossification, but not the gradual restitution of the bone to the normal. We must however remember that osteogenesis imperfecta is what its name implies, an imperfect ossification, seen in the disturbed growth at the metaphyses (Fig. 3B), and it seems reasonable to suggest that this disturbance in growth is responsible for the bone of unusual character, organized from haematomata, seen in osteogenesis imperfecta. There had never been any suspicion in my mind of sarcoma; the radiographs illustrated a benign lesion which resolved.

Baker (1946) described two similar cases under the title 'Hyperplastic Callus Simulating Sarcoma in Two Cases of Fragilitis Ossea'. One of these children falling had sustained a fracture through the femoral shaft around which a large mass formed. Five weeks later it was extensively explored for a complete biopsy. This was considered to show chondrosarcoma, but amputation was regarded as too risky and of doubtful value. Although after operation the thigh swelled excessively so that the tumour appeared to fungate through the skin, it subsided and the bony lesion resolved in the manner I have indicated. As Baker commented, 'Had the limb been removed ... .

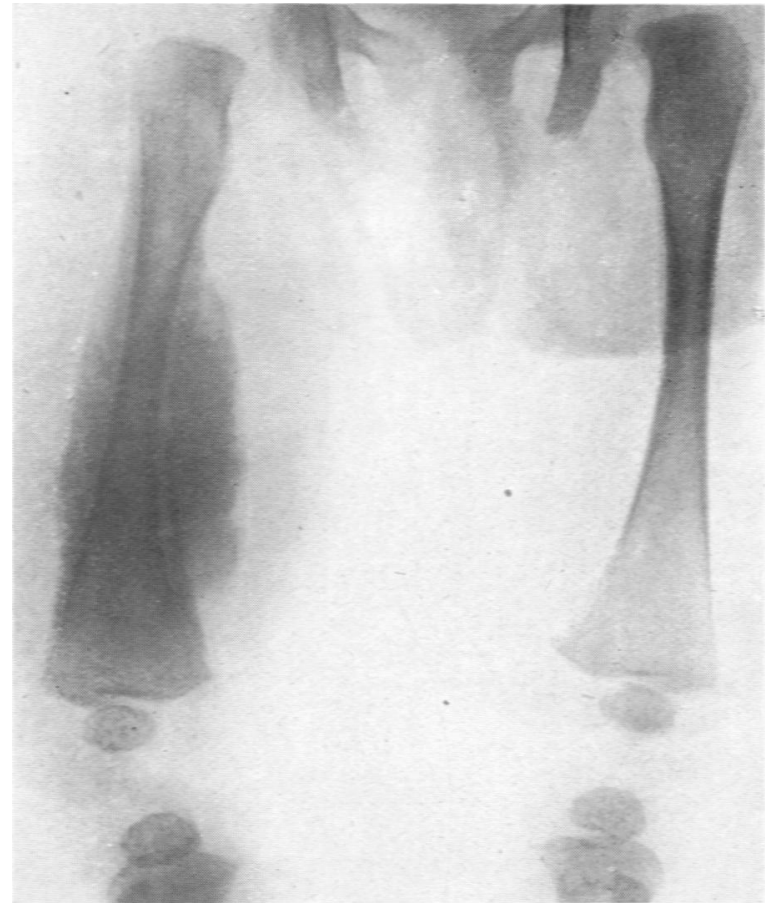

Fig. 2.-A-Radiographs of the limbs of an infant aged 3 months on August 15, 1947, with early scurvy. The right thigh shows a fusiform tumour with calcification in a large haematoma. The calcium is in an amorphous form, is ill-defined, and is separated from the cortex of the femur by a paralkel translucent zone. The diaphysis and the epiphyses of the left femur and tibia are normal in appearance.

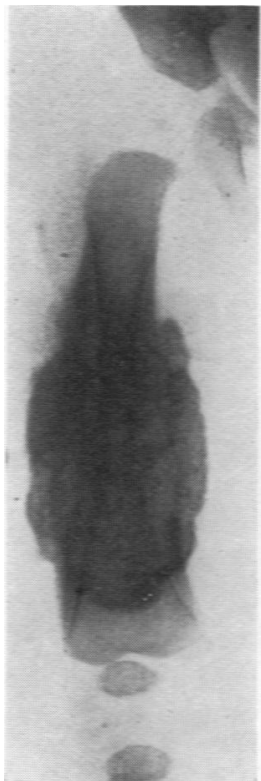

B-The right thigh on August 28, 1947, showing that some organization of the calcium has already taken place.

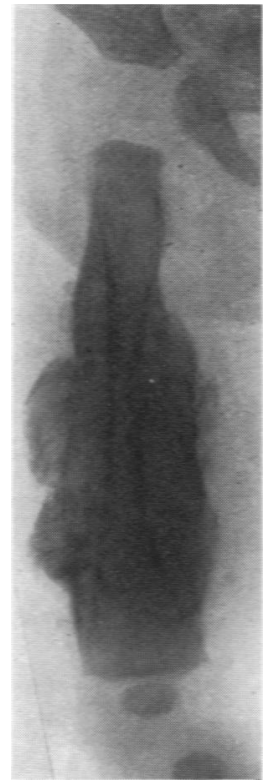

C-Same limb on September 8, 1947, after administration of vitamin $C$ had been started.

The mass has now been converted into bone with good definition.

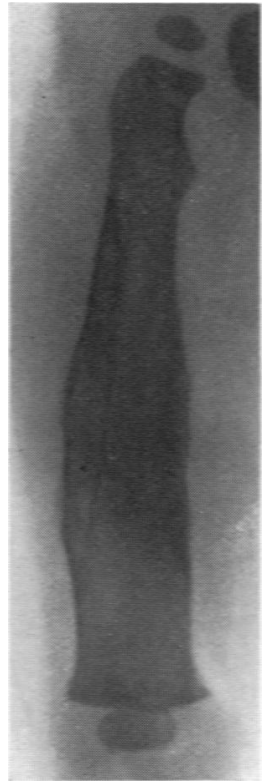

D-Same limb on January 8, 1948, when considerable resolution has taken place.

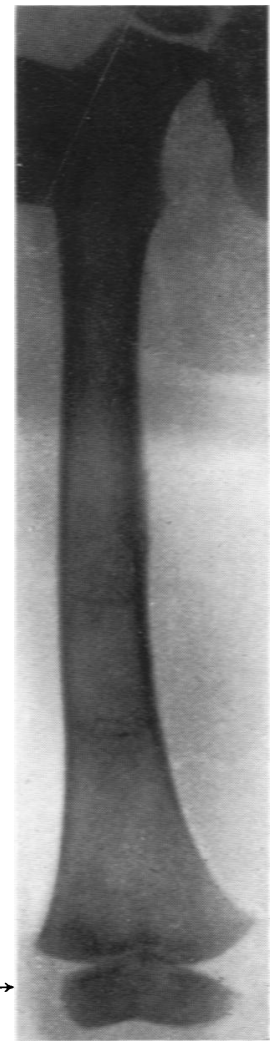




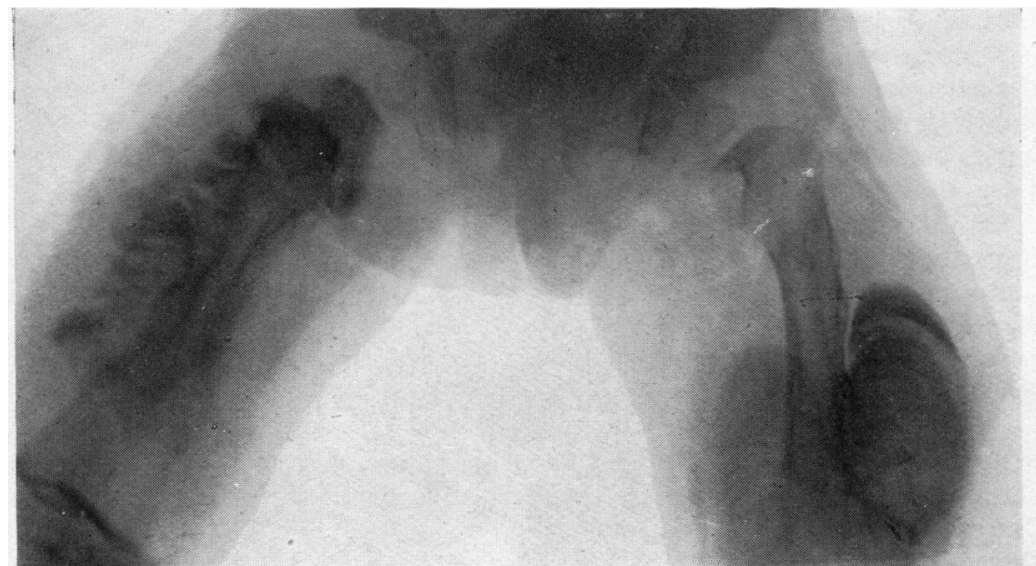

FIG. 3.-A-Radiograph on April 7, 1936, showing multiple haematomata at different ages. On the medial aspect of the femur on the right side is the most recent, on the latera aspect an older lesion undergoing organization, and on the left side the haematoma has become ossified and is shrunken.

B-The same femora three years later showing absorption of the original cortex and irregular new involucrum. Note the characteristic irregularity of osteogenesis imperfect on the borders of the metaphyses at the lower ends of the femora.

A

it would have been impossible to prove that it was not a sarcoma cured by amputation,' yet the radiographs show the sequence of changes I consider typical of haematoma. Fairbank and Baker (1948) and Watson-Jones (1952) support Baker in his revised description of the pathological condition as hyperplastic callus, although there is radiographic evidence of a similar evolution of haematomata in scurvy. Neither of these writers gives any explanation for the unusual development, nor supplies any evidence on which scurvy can be excluded, since the positive response to the therapeutic test is probably the most reliable, and probably the only supporting, evidence.

Ossifying haematomata, not only in scurvy and osteogenesis imperfecta, but also in limbs with neurovascular disturbances and in haemophilia, and even at the site of unsuspected fractures, have been mistaken from their clinical and histological evidence for sarcoma and amputation has resulted when the patients were considered to be fit enough to stand the operation. Undoubtedly some of these cases are regarded as 'cures by amputation'.

Accounts of many cases of infantile cortical hyperostoses have been published since the condition was first described but they have added little to the original description by Caffey and Silverman (1945). No adequate explanation has been put forth. It is stated by many that there is no evidence of scurvy, syphilis, or rickets and that the lesions invariably resolve without any specific treatment. It may be that the observers have looked for gross evidence and have not appreciated that scurvy may exist in certain cases before such gross evidence has been revealed. It has been indicated that it can occur without the

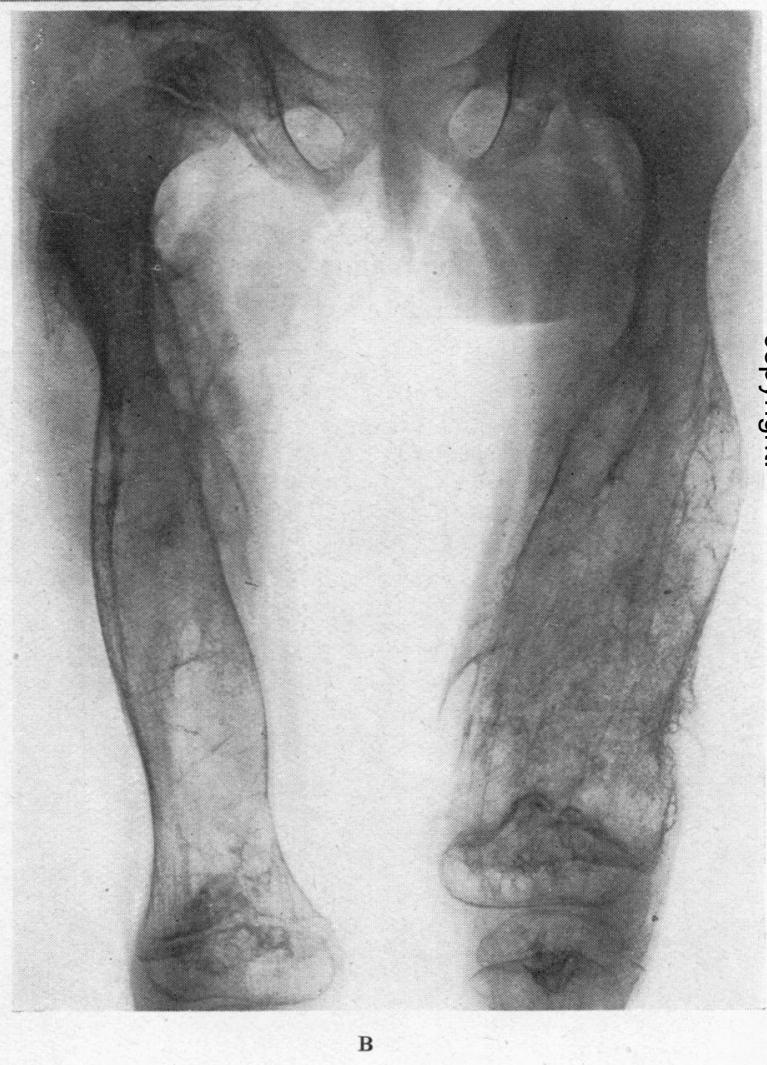

bones showing any evidence of departure from the normal texture and density, one or more fractures being the only radiographic evidence to indicate abnormality even though clinically the infant may show profound malnutrition. Fig. 1 shows the 
appearance of the bones of such an infant aged 11 months. There is no radiographic indication of scurvy though the patient was markedly emaciated and had obviously been seriously neglected. The fractures had been present for 10 to 14 days before there was any radiographic evidence of the sub-periosteal haemorrhage but it was clear that other fractures had occurred and had resolved without their presence being suspected. The rapid improvement in the clinical condition following adequate food containing fresh fruit juices and codliver oil led to the development of a fine healthy infant of nearly twice the weight within a few months. The clinical improvement was associated with calcification and ossification of the haemorrhagic foci, and within six months even the more massive periosteal masses of irregular new bone had been absorbed.

That deficiency and endocrine imbalance can be demonstrated in infants at birth we know from studies of foetal rickets and hyperparathyroidism. We know also that one of the earliest signs of scurvy is subperiosteal haemorrhage which may be so large as to be detected clinically, yet the radiographs may show apparently normal bone for a week or more, and only when sufficient calcium has been deposited to give contrast above that of the soft tissues can we see the size and position of haematomata. Certainly not until then can we detect clinically or from the radiographs subperiosteal haemorrhage of a minor degree such as could lead to the production of the radiographic appearances of cortical hyperostoses. The statement that the hyperostoses resolve with no specific medication tends to underestimate the attention which would be given to the feeding of the infants in whom these lesions have been found. This response could be regarded as the positive thera-

peutic test of scurvy. Such children would certainly be given adequate vitamins in their food; it would be unreasonable to deprive them to prove that lack of vitamins checked resolution. As will be seen from Fig. 2 the radiographic appearances of the bones are normal; there is no indication in their shape, texture, density or growth centres of scurvy; only the signs of resolution in a large haematoma indicated this. Clinically that lesion was interpreted as a sarcoma, and the radiographic evidence was thought to support this and amputation considered, but following the author's interpretation of scurvy the lesion completely resolved on the administration of vitamin C.

What is difficult to understand is the relatively sudden outburst of so many cases. Many, it would seem, develop in the U.S.A. Some isolated cases have been reported elsewhere. In Birmingham I did the radiography for all the infant welfare clinics of the city for over 20 years and never met with one such case. The suggestion could be made that such cases were not sent for radiography, but, with the prominence of the local signs, such suspicion of neglect of investigation on the part of the clinicians concerned I should regard as unreasonable, bearing in mind the triviality of the clinical signs in patients who were sent for radiography.

Multiple biopsies have been performed on many cases of infantile cortical hyperostoses but no evidence of any value for the understanding of the condition has been so obtained. Indeed biopsies could

B-Radiograph on June 1, 1949, showing the unusual ossification of the upper third of the tibia at the site of the haematoma, also more recent fractures of the lower thirds of the tibia and fibula with subperiosteal haemorrhages.

only disturb and delay complete resolution of lesions which, untouched, have been shown to resolve completely within the minimum of time. Explorations are not made before the lesions have shown considerable resolution: the evidence presented then indicates that even the nature of the lesion could not be determined. 
It may be that some deleterious factor for the foetus and infant is operating through the mother. I have evidence which indicates that the mother can transmit a 'resistance' to ordinary doses of vitamin D and calcium; further, not all the physical and chemical factors exhibited, with or without the knowledge of the doctor, during the care of the mother are necessarily beneficial to the welfare of the infant. And in any discussion of the conditions which are associated with the radiographic appearances of periosteal irregularities the effects of the administration of vitamins must be considered. I saw one infant with the typical radiographic evidence of scurvy, to whom, under medical supervision, massive doses of vitamin $\mathbf{D}$ had been given over a long period. The skeleton gradually became denser and denser until it became of the density of AlbersSchönberg's disease. The haematomata did not show the healing response seen after vitamin $C$ but persisted and developed an even greater density before the infant died.

The radiographs of infants between the ages of 6 months and 3 years, who have had excessive vitamin $A$, show some periosteal reaction and increased density at the growth extremities of the diaphyses, but, unlike cases showing infantile cortical hyperostoses, the metatarsals show periosteal accretions. Arena, Sarazen and Baylin (1951) reported hypervitaminosis $A$ in one infant of $6 \frac{1}{2}$ months. Radiographs showed craniotabes and periosteal accretions which readily resolved when administration of vitamin A ceased. Chronic poisoning due to excess vitamin $\mathbf{A}$ has been discussed and recorded by Caffey and Silverman (1945). Two of their cases showed periosteal accretions resembling the less severe cortical hyperostoses at the late age of 25 months, but no facial swelling or hyperostoses of the mandible. Seven of the patients had been given excessive amounts of vitamins $A$ and $D$ over long periods. The limbs were swollen and painful. The ribs, clavicles and long bones showed periosteal changes, but those bones which were unaffected seemed to be normal in appearance. Improvement was noted within a few days of stopping the intake of vitamins and the hyperostoses gradually resolved. (The vitamins are usually administered in oleum percomorphum which is said to contain 60,000 units of vitamin A and 8,500 units of vitamin D per g.) Such preparations are readily available in the drug stores without any need for medical supervision.
During my tour of the U.S.A. during 1948 and 1949 in the popular magazines and in the parlour cars of the trains I could not but overhear from members of the public of their common use, Indeed throughout that tour I "learnt " more in this way of allergies, infections, isotopes and the need for shots, vitamins and periodic changes in babies formulae than in any period of my medical career.

\section{Summary}

The well recognized clinical and radiographic signs of scurvy are usually not seen until the infant is 8 to 18 months of age, but the earliest radiographic signs may be those of fracture with a surrounding haematoma. In some cases such lesions may be seen without any definite clinical evidence of scurvy; in other cases emaciation and other signs of neglect may be present and yet the bones may have normal radiographic density and regularity.

The recent haematoma can be suspected from the presence of a fusiform soft-tissue swelling; the radiograph will reveal no more at this stage, but within seven to 14 days radiographic signs of the deposition of amorphous calcium can be detected towards the periphery of the haematoma. This is followed by a denser granular deposition and later its organization into bone when it shrinks and loses its regular peripheral outline. The lesion may te mistaken clinically, histologically and radiographically for sarcoma, but with vitamin $\mathrm{C}$ medication the ossified haematoma is gradually absorbed and the bone is left with normal features as distinct from the scurvy of the later age period. The radiographic features of unusual haematoma seen in certain cases of osteogenesis imperfecta and paralysed limbs and the lesions of infantile cortical hyperostoses are considered in relation to the sequence of radiographic changes in the lesions in scurvy. The best proof of scurvy may be the response to the therapeutic testcomplete resolution of the lesion when adequate vitamin $C$ is administered. The effect of vitamins on the bones is described.

\section{REFERENCES}

Arena, J. M., Sarazen, P. and Baylin, G. J. (1951). Pediatrics, 8. 788. Baker, S. L. (1946). J. Path. Bact., 58. 609.

Brailsford, J. F. (1943). Brit. J. Radiol., 16. 129

Brailsford, J. F. (1943), Brit. J. Radiol., 16, 129.

Caffey, J. and Silverman, W. A. (1945). Amer. J. Roentgenol., 54, 1.

Fairbank, H. A. T. and Baker. S. L. (1948). Brit. J. Surg., 36. 1.

Hutchison, R. and Moncrieff, A. (1941). Price's Textbook of Medicine. 6th ed., p. 454.

Watson-Jones, $\mathbf{R}$. (1952). Fractures and Joint Injuries, 4th ed. Edinburgh. 